\title{
Extremal Metrics for Laplace Eigenvalues in Perturbed Conformal Classes on Products
}

\author{
Henrik Matthiesen ${ }^{1}$ \\ Received: 8 February 2018 / Published online: 24 August 2018 \\ (c) The Author(s) 2018
}

\begin{abstract}
In this short note, we prove that conformal classes which are small perturbations of a product conformal class on a product with a standard sphere admit a metric extremal for some Laplace eigenvalue. As part of the arguments, we obtain perturbed harmonic maps with constant density.
\end{abstract}

Keywords Laplace eigenvalues · Extremal metrics · Eigenmaps

Mathematics Subject Classification 35J05 $\cdot 35 \mathrm{~J} 70 \cdot 58 \mathrm{~J} 05$

\section{Introduction}

For a closed manifold $M$, we are interested in the eigenvalues of the Laplace operator considered as functionals of the metric.

We denote by

$$
\mathcal{R}:=\{g: g \text { is a Riemannian metric on } M \text { with } \operatorname{vol}(M, g)=1\}
$$

the space of all unit volume Riemannian metrics on $M$ endowed with the $C^{\infty}$-topology, i.e. the smallest topology containing any $C^{k}$-topology. The group $C_{+}^{\infty}(M)$ of positive smooth functions acts via (normalized) pointwise multiplication on $\mathcal{R}$,

$$
\phi . g:=\operatorname{vol}(M, \phi g)^{-2 / n} \phi g
$$

so that $\operatorname{vol}(M, \phi \cdot g)=1$. The quotient space

$凶$ Henrik Matthiesen

hematt@mpim-bonn.mpg.de

1 Max Planck Institute for Mathematics, Vivatsgasse 7, 53111 Bonn, Germany 


$$
\mathcal{C}=C_{+}^{\infty}(M) \backslash \mathcal{R}
$$

is the space of all conformal structures on $M$.

Since $M$ is compact, the spectrum of $\Delta_{g}$ consists of eigenvalues of finite multiplicity only for any $g \in \mathcal{R}$. We list these as

$$
0=\lambda_{0}<\lambda_{1} \leq \lambda_{2} \leq \ldots,
$$

where we repeat an eigenvalue as often as its multiplicity requires.

In recent years, there has been much interest in finding extremal metrics for eigenvalues $\lambda_{k}$ considered either as functionals

$$
\lambda_{k}: \mathcal{R} \rightarrow \mathbb{R}
$$

or

$$
\lambda_{k}:[g] \rightarrow \mathbb{R},
$$

where

$$
[g]=\left\{\phi g: \phi \in C_{+}^{\infty}(M)\right\}
$$

denotes the conformal class of a metric $\mathrm{g}$, see for instance [4,5,7,10,11], and references therein. These functionals will not be smooth but only Lipschitz; therefore extremality has to be defined in an appropriate way, see below.

One reason to study these extremal metrics is their intimate connection to other classical objects from differential geometry. For (1.3), these are minimal surfaces in spheres, and for (1.4) these are sphere-valued harmonic maps with constant density, so called eigenmaps. There has been a lot of effort in the past to understand, which manifolds admit eigenmaps or even minimal isometric immersions into spheres, see for instance [14, Chap. 6] for a general overview over classical results for eigenmaps including the generalized Do Carmo-Wallach theorem, and $[2,8]$ to mention only the two most classical results.

Before we state our results, we have to introduce some notation. Let $M$ be a smooth, closed manifold.

A smooth map $u: M \rightarrow S^{\ell}$ is called an eigenmap, if it is harmonic, i.e.

$$
\Delta u=|\nabla u|^{2} u,
$$

and has constant density $|\nabla u|^{2}=$ const. In other words, the components of $u$ are all eigenfunctions corresponding to the same eigenvalue. Note that most Riemannian manifolds do not admit eigenmaps, since the spectrum is generically simple by [13, Theorem 8]. Even more, the spectrum of a generic metric in a conformal class is simple $[1,6,13]$. Moreover, we would like to point out that it is not clear at all whether eigenmaps exist in the presence of large multiplicity.

Theorem 1.6 Let $(M, g)$ be a closed Riemannian manifold of dimension $\operatorname{dim}(M) \geq 3$, and assume 
(i) There is a non-constant eigenmap $u:(M, g) \rightarrow S^{1}$,

or

(ii) $(M, g)=\left(N \times S^{\ell}, g_{N}+g_{s t}\right)$, where $g_{\text {st. }}$ denotes the round metric of curvature 1 on $S^{\ell}$.

Then there is a neighbourhood $U$ of $[g]$ in $\mathcal{C}$, such that for any $c \in U$, there is a representative $h \in c$, such that $(M, h)$ admits a non-constant eigenmap to $S^{1}$ respectively $S^{\ell}$.

An obvious question is then, whether the set of conformal structures admitting non-constant eigenmaps is always non-empty. We answer this at least in the following case.

Corollary 1.7 Assume $\phi: M \rightarrow S^{1}$ is a submersion. Then the set $\mathcal{E} \subset \mathcal{C}$ of conformal structures admitting non-trivial eigenmaps to $S^{1}$ is open and non-empty.

Remark 1.8 It is not clear, whether $\mathcal{E}$ is also closed. This question is related to possible degenerations of $n$-harmonic maps, as it will become clear from the proof.

Not every manifold admits a submersion to $S^{1}$. In fact, there are topological obstructions to the existence of such a map.

More precisely, since $S^{1}$ is a $K(\mathbb{Z}, 1)$, a submersion gives rise to a non-trivial element in $H^{1}(M, \mathbb{Z})$. Moreover, the differentials of local lifts of the submersion to $\mathbb{R}$ give rise to a globally defined nowhere vanishing 1-form. In particular, $M$ needs to have $\chi(M)=0$.

As mentioned above, the existence of an eigenmap $u:(M, h) \rightarrow S^{\ell}$ for a metric $h \in[g]$ implies that $h$ is extremal for some of the functionals $\lambda_{k}$ on $[g]$. Therefore, Theorem 1.6 and Corollary 1.7 have the following consequences for the existence of extremal metrics.

Corollary 1.9 Under the assumptions of Theorem 1.6, there is a neighbourhood $U$ of $[g]$ in $\mathcal{C}$, such that for any $c \in U$, there is a representative $h \in c$, such that $(M, h)$ is extremal for some eigenvalue functional on $c$.

Corollary 1.10 Under the assumptions of Corollary 1.7, the set $\mathcal{E} \subset \mathcal{C}$ of conformal structures admitting extremal metrics for some eigenvalue functional on conformal classes is open and non-empty.

The proof of Theorem 1.6 is rather simple once the correct conformally invariant formulation of the assertion is found.

This is as follows. Let $n$ be the dimension of $M$. Then a smooth map into a sphere is called $n$-harmonic, if it is a critical point of the $n$-energy

$$
E_{n}[u]=\int_{M}|\mathrm{~d} u|^{n} \mathrm{~d} V_{g},
$$

which is a conformally invariant functional. These are precisely the solutions of the equation

$$
-\operatorname{div}\left(|\nabla u|^{n-2} \nabla u\right)=|\nabla u|^{n} u .
$$


From (1.5) and (1.11), it is evident that an eigenmap defines an $n$-harmonic map, which has $\nabla u \neq 0$ everywhere. The crucial observation is that also the converse holds up to changing the metric conformally, see Lemma 3.21.

Therefore, we will be concerned with $n$-harmonic maps with nowhere vanishing derivative.

In order to deduce Corollary 1.7 from Theorem 1.6, it suffices to find a single nontrivial eigenmap $u:(M, g) \rightarrow S^{1}$ for some metric $g$. This turns out to be very easy using that $M$ is a mapping torus.

In Sect. 2, we discuss the necessary preliminaries on $n$-harmonic maps and Laplace eigenvalues. Sect. 3 contains the proofs.

\section{Preliminaries}

First, we explain the notion of extremal metrics and its connection to eigenmaps.

\subsection{Extremal Metrics for Eigenvalue Functionals}

In presence of multiplicity, the functionals $\lambda_{k}$ are not differentiable, but only Lipschitz. However, it turns out that for any analytic deformation, left and right derivatives exist. Using this, El Soufi-Ilias introduced a notion of extremal metrics for these functionals.

Definition 2.1 [3, Definition 4.1] A metric $g$ is called extremal for the functional $\lambda_{k}$ restricted to the conformal class $[g]$ of $g$, if for any analytic family of metrics $\left(g_{t}\right) \subset[g]$, with $g_{0}=g$, and $\operatorname{vol}\left(M, g_{0}\right)=\operatorname{vol}\left(M, g_{t}\right)$, we have

$$
\left.\left.\frac{\mathrm{d}}{\mathrm{d} t}\right|_{t=0^{-}} \lambda_{k}\left(g_{t}\right) \cdot \frac{\mathrm{d}}{\mathrm{d} t}\right|_{t=0+} \lambda_{k}\left(g_{t}\right) \leq 0 .
$$

We have

Theorem 2.2 [3, Theorem 4.1] The metric $g$ is extremal for some eigenvalue $\lambda_{k}$ on $[g]$ if and only if there is a eigenmap $u:(M, g) \rightarrow S^{\ell}$ given by $\lambda_{k}(g)$-eigenfunctions and either $\lambda_{k-1}(g)<\lambda_{k}(g)$, or $\lambda_{k}(g)<\lambda_{k+1}(g)$.

\subsection{Background on $n$-Harmonic Maps}

First of all, we need some background on the existence of $n$-harmonic maps. We call a map $u \in W^{1, n}\left(M, S^{\ell}\right)$ weakly $n$-harmonic, if it is a weak solution of

$$
-\operatorname{div}\left(|\nabla u|^{n-2} \nabla u\right)=|\nabla u|^{n} u .
$$

We assume that we have fixed a CW-structure on $M$, and denote by $M^{(l)}$ its $l$ skeleton. Let $v: M \rightarrow S^{\ell}$ be a Lipschitz map, where $l<n=\operatorname{dim} M$. Denote by $v^{(l)}$ the restriction of $v$ to the $l$-skeleton of $M$. The l-homotopy type of $v$ is the homotopy type of $v^{(l)}$. 
Theorem 2.4 [16, Theorem 3.4] There exists a weakly $n$-harmonic map $u: M \rightarrow$ $S^{\ell}$, with well-defined l-homotopy type, which agrees with the l-homotopy type of $v$. Moreover, $u$ minimizes the n-energy among all such maps.

We do not elaborate here on how the $l$-homotopy type is defined for maps in $W^{1, n}\left(M, S^{\ell}\right)$. For our purposes, this is not necessary, since the map $u$ is actually continuous.

Theorem 2.5 Let $u \in W^{1, n}\left(M, S^{\ell}\right)$ be a weaklyn-harmonic map, which is a minimizer for its own l-homotopy type. There is a constant $C$ depending on an upper bound on the n-energy of $u$, and on the bounds of the sectional curvature and injectivity radius of $M$, such that $\|u\|_{C^{1, \alpha}} \leq C$.

Proof Let $x \in M$ and $r>0$ be small enough. If $v \in W^{1, n}\left(B(x, r), S^{\ell}\right)$ with $u=v$ on $\partial B(x, r)$, we can consider the map $w \in W^{1, n}\left(M, S^{\ell}\right)$ given by $u$ in $M \backslash B(x, r)$ and by $v$ in $B(x, r)$. It is shown in [12, Theorem 2.8] that the $l$-homotopy type of $w$ agrees with the $l$-homotopy type of $u$. In particular, we need to have

$$
\int_{B(x, r)}|\mathrm{d} u|^{n} \mathrm{~d} V_{g} \leq \int_{B(x, r)}|\mathrm{d} v|^{n} \mathrm{~d} V_{g},
$$

which means that $u$ is a minimizing $n$-harmonic map. Therefore, the assertion follows e.g. from [9, Theorem 2.19].

In particular, these estimates are uniform as $g$ varies over a compact set of $\mathcal{R}$, as long as the energy stays bounded.

At points, in which we do not have a lack of ellipticity, we actually get higher regularity.

Theorem 2.6 A weaklyn-harmonic map $u \in C^{1, \alpha}$ is smooth near points with $\nabla u \neq 0$.

This follows from standard techniques for quasilinear elliptic equations. For completeness, we give a proof in Sect. 3.1.

The main reason for the restrictive assumptions in item $(i i)$ of Theorem 1.6 is that the above results do not imply that for a sequence $g_{k} \rightarrow g$ we can find a sequence of $n$-harmonic maps $u_{k}$ (w.r.t. $g_{k}$ ), such that $u_{k} \rightarrow u$, for a given $n$-harmonic map $u$.

In the case of maps to the circle, this problem does not appear, thanks to

Theorem 2.7 [15, Theorem A] Up to rotations of $S^{1}$, n-harmonic maps $u: M \rightarrow S^{1}$ are unique in their homotopy class.

\section{Proofs}

\subsection{Higher Regularity of $\boldsymbol{n}$-Harmonic Maps}

In this section, we give a proof of Theorem 2.6. We start with $W^{2,2}$-regularity. The proof follows using standard techniques, since under our assumptions the equation is of the form

$$
-(L u)(x)-b(x) u(x)=0,
$$


with $L$ a quasilinear operator, which is elliptic at $u$ (as demonstrated in Lemma 3.14 below) and $b \in L^{\infty}$.

Lemma 3.2 Let $U \subset M$ be open and $u:(U, g) \rightarrow S^{\ell}$ be weakly n-harmonic. Assume that $u \in C^{1, \alpha}\left(U, S^{\ell}\right)$ with $\nabla u \neq 0$ everywhere in $u$. Then we have $u \in W_{l o c}^{2,2}\left(U, S^{\ell}\right)$.

Proof For simplicity, we focus on the case $g_{i j}=\delta_{i j}$ and denote the usual differential of $u$ in Euclidean Space by $D u$. The general case follows along the same lines but with some more notation.

Take open subsets $W \subset \subset V \subset \subset U$, and a cut-off function $\eta$ which is 1 in $W$, and has $\operatorname{supp} \eta \subset V$. We show that $u \in W^{2,2}\left(W, S^{\ell}\right)$. We use the test functions given by $\phi^{k}=-D_{s}^{-h}\left(\eta^{2} D_{s}^{h} u^{k}\right)$, where $D_{s}^{h}$ denotes the difference quotient operator in coordinate direction $s$,

$$
D_{s}^{h} \phi(x)=\frac{1}{h}\left(\phi\left(x+h e_{s}\right)-\phi(x)\right) .
$$

To handle notation, let us write

$$
F_{k}^{\alpha}(D u)=|D u|^{n-2} \partial_{\alpha} u^{k}
$$

and

$$
G_{k}(u, D u)=|D u|^{n} u^{k}
$$

Then we have

$$
-\int_{U} F_{k}^{\alpha}(D u) \partial_{\alpha} D_{s}^{-h}\left(\eta^{2} D_{s}^{h} u^{k}\right)=-\int_{U} G_{k}(u, D u) D_{s}^{-h}\left(\eta^{2} D_{s}^{h} u^{k}\right)
$$

Note that this is well defined thanks to Hölder's inequality. For the left-hand side of (3.6), we have

$$
-\int_{U} F_{k}^{\alpha}(D u) \partial_{\alpha} D_{s}^{-h}\left(\eta^{2} D_{s}^{h} u^{k}\right)=\int_{U} D_{s}^{h}\left(F_{k}^{\alpha}(D u)\right) \partial_{\alpha}\left(\eta^{2} D_{s}^{h} u^{k}\right)
$$

We can write

$$
\begin{aligned}
D_{s}^{h} F_{k}^{\alpha}(D u) & =\frac{1}{h} \int_{0}^{1} \frac{\mathrm{d}}{\mathrm{d} t} F_{k}^{\alpha}\left(D u+t h D_{s}^{h} D u\right) \mathrm{d} t \\
& =\frac{1}{h} \int_{0}^{1} \frac{\partial F_{k}^{\alpha}}{\partial q_{\beta}^{l}}\left(D u+t h D_{s}^{h} D u\right) h D_{s}^{h} \partial_{\beta} u^{l} \mathrm{~d} t \\
& =\int_{0}^{1} \frac{\partial F_{k}^{\alpha}}{\partial q_{\beta}^{l}}\left(D u+t h D_{s}^{h} D u\right) \mathrm{d} t D_{s}^{h} \partial_{\beta} u^{l} \mathrm{~d} t \\
& =: \theta_{k l}^{\alpha \beta}(D u) D_{s}^{h} \partial_{\beta} u^{l} .
\end{aligned}
$$


Note that this is well defined pointwise, since $u \in C^{1, \alpha}$. The condition $|D u| \geq c>0$ implies that $\theta_{k l}^{\alpha \beta}$ are uniformly super strongly elliptic for $h \ll 1$, as demonstrated below. Since the coefficients $\theta$ are uniformly super strongly elliptic, we have

$$
\int_{U} \eta^{2}\left|D_{s}^{h} D u\right|^{2} \leq C \int_{U} \eta^{2} \theta_{k l}^{\alpha \beta}(D u)\left(D_{s}^{h} \partial_{\alpha} u^{k}\right)\left(D_{s}^{h} \partial_{\beta} u^{l}\right) .
$$

Moreover, since $\theta$ and $|D \eta|$ are bounded, we can estimate

$$
\begin{aligned}
\left|\int_{U} \theta_{k l}^{\alpha \beta}\left(D_{s}^{h} \partial_{\beta} u^{l}\right)\left(D_{s}^{h} u^{k}\right) \eta \partial_{\alpha} \eta\right| & \leq C \int_{U}\left|D_{s}^{h} D u \| D_{s}^{h} u\right| \eta \\
& \leq C \varepsilon \int_{U} \eta^{2}\left|D_{s}^{h} D u\right|^{2}+\frac{C}{\varepsilon} \int_{V}\left|D_{s}^{h} u\right|^{2} \\
& \leq C \varepsilon \int_{U} \eta^{2}\left|D_{s}^{h} D u\right|^{2}+\frac{C}{\varepsilon} \int_{U}|D u|^{2}
\end{aligned}
$$

where we have used Young's inequality and $u \in W^{1,2}$. Combining the last two estimates with (3.6) and (3.7), we find that we can choose $\varepsilon$ sufficiently small so that

$$
\int_{U} \eta^{2}\left|D_{s}^{h} D u\right|^{2} \leq C \int_{U}|D u|^{2}+C\left|\int_{U} G_{k}(u, D u) D_{s}^{-h}\left(\eta^{2} D_{s}^{h} u^{k}\right)\right|
$$

To estimate the last summand above, we note that $u,|D u| \in L^{\infty}$, implies $G_{k}(u, D u) \in$ $L^{\infty}$, and hence

$$
\begin{aligned}
\left|\int_{U} G_{k}(u, D u) D_{s}^{-h}\left(\eta^{2} D_{s}^{h} u^{k}\right)\right| & \leq C \int_{U}\left|D_{s}^{-h}\left(\eta^{2} D_{s}^{h} u^{k}\right)\right| \\
& \leq \frac{C}{\varepsilon} \operatorname{vol}(U)+C \varepsilon \int_{U}\left|D_{s}^{-h}\left(\eta^{2} D_{s}^{h} u^{k}\right)\right|^{2} \\
& \leq \frac{C}{\varepsilon}+C \varepsilon \int_{U}\left|D\left(\eta^{2} D_{s}^{h} u^{k}\right)\right|^{2} \\
& \leq \frac{C}{\varepsilon}\left(1+\int_{U}|D u|^{2}\right)+C \varepsilon \int_{U} \eta^{2}\left|D_{s}^{h} D u^{k}\right|^{2} .
\end{aligned}
$$

For $\varepsilon$ sufficiently small, we can absorb the last term, and find

$$
\int_{V}\left|D_{s}^{h} D u\right|^{2} \leq \int_{U} \eta^{2}\left|D_{s}^{h} D u\right|^{2} \leq \frac{C}{\varepsilon}\left(1+\int_{U}|D u|^{2}\right) .
$$

Thus $u \in W_{l o c}^{2,2}\left(U, S^{\ell}\right)$.

We still need to justify that the coefficients $\theta_{k l}^{\alpha \beta}$ are uniformly super strongly elliptic.

Lemma 3.14 There is $h_{0}>0$ depending on $\|D u\|_{C^{0, \alpha}}$ such that we have $\theta_{k l}^{\alpha \beta} A_{\alpha}^{k} A_{\beta}^{l} \geq$ $v|A|^{2}$, for any $h$ with $|h| \leq h_{0}$ and $v=v(c)$, where $|D u|^{2} \geq c$. 
Proof We have

$$
\frac{\partial F_{k}^{\alpha}}{\partial q_{\beta}^{l}}(q)=|q|^{n-4}\left(|q|^{2} \delta^{\alpha \beta} \delta_{k l}+(n-2) q_{\alpha}^{k} q_{\beta}^{l}\right) .
$$

Thus, it is not very hard to see that

$$
\begin{aligned}
\frac{\partial F_{k}^{\alpha}}{\partial q_{\beta}^{l}}(q) A_{\alpha}^{k} A_{\beta}^{l} & =|q|^{n-4}\left(|q|^{2} \delta^{\alpha \beta} \delta_{k l} A_{\alpha}^{k} A_{\beta}^{l}+(n-2) q_{\alpha}^{k} q_{\beta}^{l} A_{\alpha}^{k} A_{\beta}^{l}\right) \\
& \geq|q|^{n-2}|A|^{2} \\
& \geq 2 v|A|^{2},
\end{aligned}
$$

as long as $|q|^{2} \geq(2 v)^{2 /(n-2)}$. Since $D u \in C^{0, \alpha}$, and $|D u|^{2} \geq c$, we can choose $h_{0} \ll 1$, such that $\left|(1-t) D u\left(x+h e_{s}\right)+t D u(x)\right|^{2} \geq c / 2$, for all $x$, and $|h| \leq h_{0}$. Clearly, this implies

$$
\begin{aligned}
\theta_{k l}^{\alpha \beta}(D u)(x) A_{\alpha}^{k} A_{\beta}^{l} & =\int_{0}^{1} \frac{\partial F_{k}^{\alpha}}{\partial q_{\beta}^{l}}\left((1-t) D u\left(x+h e_{s}\right)+t D u(x)\right) A_{\alpha}^{k} A_{\beta}^{l} \mathrm{~d} t \\
& \geq \int_{0}^{1} v|A|^{2} \mathrm{~d} t \\
& \geq v|A|^{2}
\end{aligned}
$$

for $v=c^{(n-2) / 2} / 2$.

In the next step, we derive the equation for $\partial_{\alpha} u^{k}$ and apply Schauder estimates to gain higher regularity. In particular, this completes the proof of Theorem 2.6

Lemma 3.17 Under the above assumptions, the function $u$ is smooth.

Proof Write

$$
\vartheta_{k l}^{\alpha \beta}=\frac{\partial F_{k}^{\alpha}}{\partial q_{\beta}^{l}} .
$$

By the calculation above, these coefficients are uniformly super strongly elliptic at $u$. We test the equation for $u^{k}$ with $\partial_{\alpha} \phi^{k}$ for some test function $\phi$ and integrate by parts in order to find

$$
\int_{U} \vartheta_{k l}^{\alpha \beta}(\nabla u) \partial_{\beta \gamma} u^{l} \partial_{\alpha} \phi^{k}=\int_{U} \partial_{\gamma} G_{k}(u, \nabla u) \phi^{k} .
$$

In other words, $v=\partial_{\gamma} u$ is a weak solution to

$$
-\operatorname{div}(\vartheta(D u) v)=\partial_{\gamma} G(u, D u)
$$

Since $|D u|^{2} \geq c>0$, the right-hand side of this equation is in $C^{k, \alpha}$, once we have $u \in C^{k+1, \alpha}$. In this case, the left-hand side has coefficients in $C^{k, \alpha}$; thus, it follows 
that $v \in C^{k+1, \alpha}$ and thus $u \in C^{k+2, \alpha}$. Since we know $u \in C^{1, \alpha}$, we can start this bootstrap argument at $k=0$, and get $u \in C^{\infty}$.

\subsection{Proofs of Main Results}

We start with the following simple but crucial observation.

Lemma 3.21 Let $u:(M, g) \rightarrow S^{\ell}$ be a smooth $n$-harmonic map with $\mathrm{d} u \neq 0$ everywhere. Then there is metric $g^{\prime}$ conformal to $g$, such that $u:\left(M, g^{\prime}\right) \rightarrow S^{\ell}$ is an eigenmap.

Proof Define $g^{\prime}=|\mathrm{d} u|_{g}^{2} g$. Since we assumed $\mathrm{d} u \neq 0$ everywhere, this defines a smooth metric, which is conformal to $g$. Then $|\mathrm{d} u|_{g^{\prime}}^{2}=\left|\mathrm{d} u_{g}\right|^{-2}|\mathrm{~d} u|_{g}^{2}=1$. Finally, $u$ solves

$$
-\operatorname{div}_{g}\left(|\mathrm{~d} u|_{g}^{n-2} \nabla u\right)=|\mathrm{d} u|_{g}^{n} u
$$

which can also be written as

$$
\Delta_{g^{\prime}} u=-\frac{1}{|\mathrm{~d} u|_{g}^{n}} \operatorname{div}_{g}\left(|\mathrm{~d} u|_{g}^{n-2} \nabla u\right)=u,
$$

and hence $u:\left(M, g^{\prime}\right) \rightarrow S^{\ell}$ is an eigenmap.

In order to prove Theorem 1.6, it now suffices to show that metrics close to the initial metric $g$ on $M$ also admit smooth $n$-harmonic maps with nowhere vanishing derivative.

Proof of Theorem 1.6 (i) Let $u:(M, g) \rightarrow S^{1}$ be an eigenmap and assume that the assertion of the theorem was not correct. This means that any neighbourhood $U \subset \mathcal{C}$ of $[g]$ contains a conformal class which does not contain any representative which admits an eigenmap to $S^{1}$. Let $U_{k} \subset \mathcal{R}$ be a sequence of open neighbourhoods of $g$ with $\cap_{k \in \mathbb{N}} U_{k}=\{g\}$. (Such a sequence exists since the $C^{\infty}$-topology on $\mathcal{R}$ is first countable and Hausdorff.) Denote by $\pi: \mathcal{R} \rightarrow \mathcal{C}$ the quotient map and observe that this is an open map. In particular, the sets $\pi\left(U_{k}\right) \subset \mathcal{C}$ are open and we can find $g_{k} \in U_{k}$ such that no metric in $\left[g_{k}\right]$ admits an eigenmap to $S^{1}$. By Lemma 3.21, this implies that $g_{k}$ itself cannot admit a nowhere vanishing $n$-harmonic map to $S^{1}$.

We now plan to use Theorem 2.4 to obtain weakly $n$-harmonic maps $u_{k}:\left(M, g_{k}\right) \rightarrow$ $S^{1}$ which are close to $u$ for $k$ sufficiently large. By assumption, the $u_{k}$ have some point $x_{k}$ with $\mathrm{d} u_{k}\left(x_{k}\right)=0$. This forces $u$ to have a critical point as well, which gives the desired contradiction.

More precisely, we apply Theorem 2.4 to $u:\left(M, g_{k}\right) \rightarrow S^{1}$ and obtain $n$-harmonic representatives $u_{k}:\left(M, g_{k}\right) \rightarrow S^{1}$ of $[u]$. If $\mathrm{d} u_{k} \neq 0$ everywhere, Theorem 2.6 implies that $u_{k}$ is a smooth $n$-harmonic map from $\left(M, g_{k}\right)$ to $S^{1}$ with nowhere vanishing derivative contradicting the construction of $g_{k}$ in the preceding paragraph. Therefore, we can find $x_{k} \in M$ such that $\mathrm{d} u_{k}\left(x_{k}\right)=0$. Since $\operatorname{dim}(M) \geq 3$ and $S^{1} \simeq K(\mathbb{Z}, 1)$, we have that $w \simeq u$ if and only if their $l$-homotopy type agrees for some $l \geq 2$. In particular, we have that 


$$
\int_{M}\left|\mathrm{~d} u_{k}\right|^{n} \mathrm{~d} V_{g_{k}} \leq \int_{M}|\mathrm{~d} u|^{n} \mathrm{~d} V_{g_{k}} \leq C \int_{M}|\mathrm{~d} u|^{n} \mathrm{~d} V_{g},
$$

so that we are in the position to apply Theorem 2.5.

By taking a subsequence if necessary, we may assume that $x_{k} \rightarrow x$. Thanks to Theorem 2.5 and the compact embedding $C^{1, \alpha}(M) \hookrightarrow C^{1, \beta}(M)$ for $\beta<\alpha$, we can extract a further subsequence, such that $u_{k} \rightarrow v$ in $C^{1, \beta}(M, g)$. We have

$$
\begin{aligned}
\int_{M}|\mathrm{~d} v|_{g} \mathrm{~d} V_{g} & =\lim _{k \rightarrow \infty} \int_{M}|\mathrm{~d} v|_{g_{k}} \mathrm{~d} V_{g_{k}} \\
& \leq \lim _{k \rightarrow \infty}\left(\int_{M}\left|\mathrm{~d} u_{k}\right|_{g_{k}} \mathrm{~d} V_{g_{k}}+\left.\int_{M}|| \mathrm{d} v\right|_{g_{k}}-\left|\mathrm{d} u_{k}\right|_{g_{k}} \mid \mathrm{d} V_{g_{k}}\right) \\
& \leq \lim _{k \rightarrow \infty}\left(\int_{M}|\mathrm{~d} w|_{g_{k}} \mathrm{~d} V_{g_{k}}+C d_{C^{1, \beta}\left(M, g_{k}\right)}\left(v, u_{k}\right)\right) \\
& \leq \lim _{k \rightarrow \infty} \int_{M}|\mathrm{~d} w|_{g_{k}} \mathrm{~d} V_{g_{k}}+\lim _{k \rightarrow \infty} C d_{C^{1, \beta}(M, g)}\left(v, u_{k}\right) \\
& =\int_{M}|\mathrm{~d} w|_{g} \mathrm{~d} V_{g},
\end{aligned}
$$

for any $w \simeq u$. It follows that $v$ is $n$-harmonic and homotopic to $u$. Thus, it follows from Theorem 2.7 that there is $A \in S O(2)$, such that $A \circ v=u$. Then $A \circ u_{k} \rightarrow u$ in $C^{1, \beta}(M)$. It follows that

$$
|\mathrm{d} u(x)| \leq \limsup _{k \rightarrow \infty} C d\left(x, x_{k}\right)^{\beta}=0,
$$

contradicting the assumption on $u$.

In order to adapt the strategy from above for more general situations, we need to understand whether there exist eigenmaps $u:(M, g) \rightarrow S^{\ell}$, which can be approximated through $n$-harmonic maps for any sequence of metrics $g_{k} \rightarrow g$.

This is precisely what we do now for product metrics $g_{s t}+g_{N}$ on $S^{\ell} \times N$. The natural candidate here is the projection map onto $S^{\ell}$. In what follows $n$ will denote the dimension of $N$, so that the dimension of $N \times S^{\ell}$ is $n+l$.

Proposition 3.22 Let $g=g_{N}+g_{s t}$. be a product metric on $N \times S^{\ell}$, with $g_{s t}$, the round metric of curvature 1 on $S^{\ell}$. The projection $u: N \times S^{\ell} \rightarrow S^{\ell}$ onto the second factor is the unique minimizer for the $(n+l)$-energy in its l-homotopy class up to rotations of $S^{\ell}$.

Proof Let $v: N \times S^{\ell} \rightarrow S^{\ell}$ be a Lipschitz map whose restriction to the $l$-skeleton of $N \times S^{\ell}$ is homotopic to the restriction of the projection $N \times S^{\ell} \rightarrow S^{\ell}$ to the $\ell$-skeleton. We want to estimate

$$
\int_{N \times S^{\ell}}|\mathrm{d} v|_{g}^{n+\ell} \mathrm{d} V_{g}
$$

from below. 
We have

$$
\begin{aligned}
& \int_{N \times S^{\ell}}|\mathrm{d} v|_{g}^{n+\ell} \mathrm{d} V_{g} \\
& \quad=\int_{N} \int_{S^{\ell}}\left(\left|\nabla^{N} v\right|^{2}+\left|\nabla^{S^{\ell}} v\right|^{2}\right)^{(n+\ell) / 2}(x, \theta) \mathrm{d} \theta \mathrm{d} x \\
& \geq \int_{N} \int_{S^{\ell}}\left|\nabla^{S^{\ell}} v\right|^{n+\ell}(x, \theta) \mathrm{d} \theta \mathrm{d} x \\
&\left.\geq(\ell+1) \omega_{\ell+1}\right)^{-n / \ell} \int_{N}\left(\int_{S^{\ell}}\left|\nabla^{S^{\ell}} v\right|^{\ell}(x, \theta) \mathrm{d} \theta\right)^{(n+\ell) / \ell} \mathrm{d} x,
\end{aligned}
$$

where we have used Hölder's inequality in the last step. Equality holds in the above inequalities if and only if $\left|\nabla^{M} v\right|^{2}=0$ and $\left|\nabla^{S^{\ell}} v\right|^{2}=$ const.

In order to estimate the remaining integral in the last line of (3.24), we use that the maps $v(x, \cdot): S^{\ell} \rightarrow S^{\ell}$ have degree 1 . This can be seen by inspecting the $l$-homotopy type of $v$ : If we endow $S^{\ell}$ with the $\mathrm{CW}$-structure consisting of a single 0 - and a single $\ell$-cell, we have $\left(N \times S^{\ell}\right)^{(\ell)}=N^{(\ell)} \times\left\{\theta_{0}\right\} \cup\left\{x_{0}\right\} \times S^{\ell}=N^{(\ell)} \vee S^{\ell}$ with $\theta_{0} \in S^{\ell}$ and $x_{0} \in N$ corresponding to the 0 -cells. The projection onto $S^{\ell}$ restricts to the map $N^{(\ell)} \vee S^{\ell} \rightarrow S^{\ell}$ that collapses the first summand and is the identity on $S^{\ell}$. In particular, we find that for any $v$, such that $v^{(\ell)}$ is homotopic to the map described above, the degree of $v\left(x_{0}, \cdot\right): S^{\ell} \rightarrow S^{\ell}$ equals 1 . Since $N$ is connected, $v(x, \cdot) \simeq v\left(x_{0}, \cdot\right)$ for any $x$, thus $\operatorname{deg} v(x, \cdot)=1$ for any $x \in N$.

This implies that

$$
\int_{S^{\ell}}\left|\nabla^{S^{\ell}} v\right|^{l}(x, \theta) \mathrm{d} \theta \geq(\ell+1) \omega_{\ell+1} \ell^{\ell / 2}|\operatorname{deg} v(x, \cdot)|=(\ell+1) \omega_{\ell+1} l^{\ell / 2} .
$$

Here, equality holds if and only if $v(x, \cdot)$ is conformal. Combining (3.24) and (3.25), we find

$$
\int_{N \times S^{\ell}}|\mathrm{d} v|_{g}^{n+\ell} \mathrm{d} V_{g} \geq \operatorname{vol}(N)(\ell+1) \omega_{\ell+1} l^{(n+\ell) / 2},
$$

with equality if and only if $\left|\nabla^{M} v\right|^{2}=0$, and $\left|\nabla^{S^{\ell}} v\right|^{2}=$ const., and $v(x, \cdot)$ is conformal. It follows in this case that $v(x, \theta)=\tilde{v}(\theta)$ with $\tilde{v}: S^{\ell} \rightarrow S^{\ell}$ of degree 1. Observe that $u: M \times S^{\ell} \rightarrow S^{\ell}$ realizes the equality in (3.26). Therefore,

$$
\inf _{v} \int_{N \times S^{\ell}}|\mathrm{d} v|^{n+\ell} \mathrm{d} V_{g}=\operatorname{vol}(N)(\ell+1) \omega_{\ell+1} l^{(n+\ell) / 2},
$$

where the infimum is taken over all Lipschitz maps $v$ having the $l$-homotopy of $u$. In particular, by the equality discussion above, minimizers need to be $(n+l)$-harmonic maps $v(x, \theta)=\tilde{v}(\theta)$, with $|\nabla v|^{2}=$ const. Therefore, $\tilde{v}$ defines a harmonic selfmap of $S^{\ell}$ with constant density. Since $\tilde{v}$ is non-trivial, it follows that $|\nabla \tilde{v}|^{2} \geq \lambda_{1}\left(S^{\ell}\right)=l$. Consequently, equality in (3.26) is only achieved by maps of the form $A \circ u$, with $A \in O(l+1)$. 
Using Proposition 3.22 instead of Theorem 2.7, assertion (ii) of Theorem 1.6 follows along the same lines as assertion $(i)$.

Proof of Corollary 1.7 Let $f: M \rightarrow S^{1}$ be a submersion. Since $M$ is compact this is a proper submersion. Moreover, $f$ has to be surjective, since otherwise $M$ would be contractible. It follows by Ehresmann's lemma that $f: M \rightarrow S^{1}$ is a fibre bundle, $F \rightarrow M \rightarrow S^{1}$, with $F$ a smooth $(n-1)$-dimensional manifold. As a consequence, there is a diffeomorphism $\phi: F \rightarrow F$, such that $M$ is obtained as the mapping torus corresponding to $\phi$, i.e.

$$
M \cong(F \times[0,1]) /(x, 0) \sim(\phi(x), 1) .
$$

Choose a metric $g_{0}$ on $F$, which is invariant under $\phi$. We claim that the metric $g_{1}=$ $g_{0}+\mathrm{d} t^{2}$ defined on $F \times[0,1]$ descends to a smooth metric $g$ on $M$. Clearly, $g_{1}$ descends to a metric $g$ on $M$, we only need to check that it is smooth. This is clear near all points $(x, t)$ with $t \neq 0,1$. We have coordinates with values in $F \times(-\varepsilon, \varepsilon)$ near the $t=0$-slice as follows:

$$
(x, t) \mapsto \begin{cases}(x, t-1) & \text { if } t \leq 1 \\ (\phi(x), t) & \text { if } t>0 .\end{cases}
$$

In these coordinates, $g$ is given by $g_{0}+\mathrm{d} t^{2}$, since $g_{0}$ is $\phi$-invariant.

It remains to show that $(M, g)$ admits an eigenmap. Define $u: F \times[0,1] /(x, 0) \sim$ $(f(x), 1) \rightarrow S^{1}$ by $(x, t) \mapsto t$. With respect to $g$ this is a Riemannian submersion. Moreover, it follows from (3.28) that $u$ has totally geodesic fibres. Thus $u$ is an eigenmap.

Acknowledgements It is a pleasure to thank my advisor Werner Ballmann for permanent support. I gratefully acknowledge the support and hospitality of the Max Planck Institute for Mathematics in Bonn. I would also like to thank the referee for many comments that helped to significantly improve the presentation. Open access funding provided by Max Planck Society.

Open Access This article is distributed under the terms of the Creative Commons Attribution 4.0 International License (http://creativecommons.org/licenses/by/4.0/), which permits unrestricted use, distribution, and reproduction in any medium, provided you give appropriate credit to the original author(s) and the source, provide a link to the Creative Commons license, and indicate if changes were made.

\section{References}

1. Bleecker, D.D., Wilson, L.C.: Splitting the spectrum of a Riemannian manifold. SIAM J. Math. Anal. 11(5), 813-818 (1980)

2. Bryant, R.L.: Conformal and minimal immersions of compact surfaces into the 4-sphere. J. Differ. Geom. 17(3), 455-473 (1982)

3. El Soufi, A., Ilias, S.: Laplacian eigenvalue functionals and metric deformations on compact manifolds. J. Geom. Phys. 58(1), 89-104 (2008)

4. El Soufi, A., Giacomini, H., Jazar, M.: A unique extremal metric for the least eigenvalue of the Laplacian on the Klein bottle. Duke Math. J. 135(1), 181-202 (2006)

5. Fraser, A., Schoen, R.: Sharp eigenvalue bounds and minimal surfaces in the ball. Invent. Math. 203(3), 823-890 (2016) 
6. Guillemin, V., Legendre, E., Sena-Dias, R.: Simple spectrum and Rayleigh quotients. Geom. Spectr. Anal. 630, 33-44 (2014)

7. Kokarev, G.: Variational aspects of Laplace eigenvalues on Riemannian surfaces. Adv. Math. 258, 191-239 (2014)

8. Lawson Jr, H.B.: Complete minimal surfaces in $S^{3}$. Ann. Math. (2) 92, 335-374 (1970)

9. Naber, A., Valtorta, D., Veronelli, G.: Quantitative regularity for p-harmonic maps (2014). arXiv: 1409.8537

10. Nadirashvili, N.: Berger's isoperimetric problem and minimal immersions of surfaces. Geom. Funct. Anal. 6(5), 877-897 (1996)

11. Petrides, R.: Existence and regularity of maximal metrics for the first Laplace eigenvalue on surfaces. Geom. Funct. Anal. 4(4), 1336-1376 (2014)

12. Pigola, S., Veronelli, G.: On the Dirichlet problem for $p$-harmonic maps I: compact targets. Geom. Dedicata 177, 307-322 (2015)

13. Uhlenbeck, K.: Generic properties of eigenfunctions. Am. J. Math. (9) 98, 1059-1078 (1976)

14. Urakawa, H.: Calculus of Variations and Harmonic Maps. Translations of Mathematical Monographs, vol. 132. American Mathematical Society, Providence (1993)

15. Veronelli, G.: A general comparison theorem for $p$-harmonic maps in homotopy classes. J. Math. Anal. Appl. 391(2), 335-349 (2012)

16. White, B.: Homotopy classes in Sobolev spaces and the existence of energy minimizing maps. Acta Math. 160(1-2), 1-17 (1988) 